http://jmscr.igmpublication.org/home/ ISSN (e)-2347-176x ISSN (p) 2455-0450 crossref DOI: https://dx.doi.org/10.18535/jmscr/v8i1.83

\title{
Retrospective Analysis of Curcumin in Cervical Cancer Patients Post Radiotherapy
}

\author{
Authors \\ Nisarga Vontikoppal Manjunatha ${ }^{1}$, Divyashree Shivalingaiah Jayashankar ${ }^{1}$, \\ Harshitha Kesargere Ramesh ${ }^{1}$, Anu Kodihalli Raju1, Vijayalaxmi Patil ${ }^{1}$, \\ Lokesh Vishwanath ${ }^{2}$, Naveen Thimmiah ${ }^{3}$, Sridhar Poojar ${ }^{4}$, Vijay Calmuge Raghu ${ }^{5}$ \\ ${ }^{1}$ Post Graduate Student, Department of Radiation Oncology, Kidwai Memorial Institute of Oncology \\ Bangalore-29 \\ ${ }^{2}$ Professor and Head of Department, Department of Radiation Oncology, Kidwai Memorial Institute of \\ Oncology, Bangalore-29 \\ ${ }^{3}$ Professor, Department of Radiation Oncology, Kidwai Memorial Institute of Oncology, Bangalore-29 \\ ${ }^{4}$ Assistant Professor, Department of Radiation Oncology, Kidwai Memorial Institute of Oncology \\ Bangalore-29 \\ ${ }^{5}$ Assistant Professor, Department of Epidemiology and Biostatistics, Kidwai Memorial Institute of \\ Oncology, Bangalore-29 \\ *Corresponding Author
}

Anu Kodihalli Raju

Post Graduate, Department of Radiation Oncology, Kidwai Memorial Institute of Oncology, Bengaluru-29

Abstract
Aim of the Study: To Evaluate the Effect of Curcumin on Toxicity and Outcomes in Cervical Cancer
patients Post Radiotherapy.
Material and Methods: 60 Cervical Cancer patients who received definitive Chemo radiation and
Brachytherapy were selected for the study. Patients received External beam Radiotherapy by 3DCRT
technique to a total dose of $45-50 G Y$ with concurrent Platinum based chemotherapy of 5 cycles and
Brachytherapy was delivered by HDR technique, limiting the Bladder and Rectal doses to $75-80 G Y$ and
$70-75 G Y$ respectively.
Of these, 30 Patients received Tab. Curcumin 500mg BID for a minimum period of 3 months post
Radiotherapy during Follow-up and were compared with patients of similar characteristics kept only
under Observation. The toxicity and outcomes post Radiotherapy was analyzed. The incidence of
Radiation Dermatitis, Radiation Cystitis and Radiation Proctitis was analyzed.
Results: At 3 months post Radiotherapy, Radiation Dermatitis was seen in 20 out of 30 patients in the
observation arm with Grade I toxicity in 12 patients, Grade II toxicity in 6 patients and Grade III toxicity
in 2 patients compared tol2 patients having Grade I toxicity who received Curcumin (p=0.038) which
was significant. Radiation Cystitis was seen in 21 patients in the observation arm with Grade I Toxicity in
16 Patients, Grade II Toxicity in 4 Patients and Grade IV Toxicity in 1 patientcompared to 12 patients with
Grade I Cystitis in Curcumin arm (p=0.0198) which was significant. Radiation Proctitis was seen in 23
patients in the observation arm with Grade I toxicity in 14 Patients and Grade II Toxicity in 9 patients
compared to 8 patients with Grade I proctitis and 1 patient with Grade II toxicity in Curcumin arm


$(p=0.001)$ which was significant. There was no evidence of disease in 25 patients and only 5 patients had Stable disease in Curcumin arm compared to 6patients with Stable disease and 4patients with Progressive disease and no evidence of disease was seen in 20 patients in Observation arm.

Conclusions: Radiation Dermatitis, Cystitis and Proctitis are well known complications of radiation treatment in Carcinoma Cervix patients. Early detection and treatment reduces the grade of toxicity and improves Quality of life. The use of Curcumin post radiation definitely reduces the side effects and thereby improves the therapeutic ratio. Better local control with Curcumin must also be proved with large randomized control trials addressing all the bias. With the advent of nano-curcumin there is better absorption and bio- availibility for enhanced effects of curcumin and improving the quality of life. The optimal dosage and duration of curcumin post radiotherapy to be administered is still investigational.

Keywords: Curcumin, Dermatitis, Cystitis, Proctitis, Cervical Cancer, Radiotherapy.

\section{Introduction}

Cervical Cancer represents the $4^{\text {th }}$ most common malignancy among women worldwide ${ }^{[1]}$. But, it's the second most common cancer in India accounting for $22.86 \%$. In Karnataka, it ranks number- 1 disease among women from rural areas, accounting for $23 \%$ of the cases being registered at our institute (HBCR KMIO- 2015) ${ }^{[2]}$. More than $60 \%$ of patients present to our Institute in advanced stage. Attaining good Local control rates and better Overall survival rates still remains a challenge even in this modern era of Advanced treatment.

Radiotherapy with concurrent Chemotherapy still remains the standard of care treatment for Locally advanced Cancer Cervix. Cervical Cancer is a potentially Radio curable disease .Our Institute's policy is to treat carcinoma cervix with Platinum based Chemotherapy during External beam radiotherapy (National Cancer Institute alert, publication February-1999), followed by Brachytherapy. The Brachytherapy is an integral component of Standard treatment protocol, incorporation of which along with External Beam radiotherapy has shown good local control with significant survival improvement. Minimizing the Bladder and Rectal doses and thereby reducing the incidences of toxicities remains a challenge even with 3D-Image based Radiotherapy.

In the Indian Culture, "Turmeric" plays a pivotal role in terms of customs and food habits. The usage of Turmeric dates back to Vedic period. Turmeric is used for a wide variety of ailments and conditions in our day to day Life, so it has become "Spice of Life". Curcumin is a pigment in the rhizomes of turmeric, which gives yellow color and adds the flavor to dishes. It has antioxidant, anti-inflammatory, anti-viral, antibacterial, anti-fungal and anti-cancer effects. There are various chemical components in turmeric like Curcumin (Diferuloyl methane), DMethyoxy-Curcumin (DMC), Bis-D-MethoxyCurcumin (BDMC), Tetrahydro Curcumin (THC) and Turmerones.

Curcumin exhibit the properties of Tumor Necrosis Factor (TNF) Blocker, Vascular Endothelial Growth Factor (VEGF) Blocker, Human Epidermal Growth Factor Receptor (hEGFR) blocker. Of the components derived from turmeric, Curcumin was found to be the most active ingredient for its ability to suppress TNFAlfa induced NFk-beta activation. It also suppresses the proliferation of Leukemic, Prostate and Breast cancer cells. It also suppresses inflammatory pathways and cellular proliferation by NF-K beta, TNF-alfa, STAT-3 pathways ${ }^{[3]}$.

Curcumin is inhibitor of NF-k beta for treatment of human Myeloid ML1-alfa cells with TNF rapidly activated NF-Alfa beta, which consists of p50,p65 subunits and this activation was inhibited by curcumin. Activator protein-1(AP-1) binding factors were also found to be down-regulated by curcumin, whereas the SP-1 binding factor was unaffected. Besides TNF-Alfa, curcumin also blocked phorbol ester and hydrogen peroxide mediated activation of NF-k beta ${ }^{[4]}$.

Curcumin also inhibits Cox-2 and Cyclin-D1 protein expression in a dose dependent manner. It can be used from 1-6g/day and maximum ceiling dose of curcumin is not known. Curcumin inhibits 
STAT3 (signal transducer and activator of transcription 3). It inhibits IL-6 induced STAT3 phosphorylation and consequent nuclear translocation. It also inhibits IF-Alfa induced signal transducer and activator of transcription-1 phosphorylation. Curcumin inhibits TNF-Alfa, which is a cell signaling cytokine, which is associated with systemic inflammatory process in the body. All the above effects of curcumin makes it a potent drug to minimize toxicities ${ }^{[5]}$.

\section{Pathogenesis}

Radiation dermatitis is caused due to Proinflammatory cytokines(IL-3,5,6 and TNF-Alfa), Chemokines (exotaxin and IL-8), Receptor Tyrosine Kinase and Adhesion Molecules (ICAM1, E-Selectin and Vascular Cell Adhesion proteins) which causes local inflammatory reaction, leading to tissue damage ${ }^{[6]}$.

The incidence of Radiation Cystitis is bladder dose dependent. Radiation causes double strand breaks resulting in DNA damage and cell death. The irreparably damaged DNA which is formed due to irradiation causes endarteritis obliterans and ischemia of the Bladder tissue resulting in reduced nutrition leading to fibrosis and necrosis. The time duration of this process is not precisely delineated because the damaged blood vessels can survive from months to years of injury, thus making it difficult to predict the time frame of early detection $^{[7]}$.

Development of Radiation Proctitis is also dose dependent, which varies between 2-39\% based on dose received to rectum and irradiation technique and brachytherapy techniques. Pathogenesis of radiation proctitis is found to be due to progressive epithelial atrophy, submucosal fibrosis, ischemia due to endarteritis leading to fragile neovascularization ${ }^{[8]}$.

\section{Material and Methods}

60 Cervical Cancer patients who received definitive Chemo radiation and Brachytherapy were selected for the study. Patients received
External beam Radiotherapy by 3DCRT technique to a total dose of 45-50GY with concurrent Platinum based chemotherapy of 5cycles and Brachytherapy was delivered by High dose rate (HDR) technique limiting the Bladder and Rectal doses to 75-80GY and 70-75GY respectively.

Of these, 30 Patients received Tab. Curcumin $500 \mathrm{mg}$ BID for a minimum period of 3 months post Radiotherapy during Follow-up and were compared with patients of similar characteristics kept only under Observation. The toxicity and outcomes post Radiotherapy was analyzed. The incidence of Radiation Dermatitis, Radiation Cystitis and Radiation Proctitis were analyzed.

\section{Results}

Statistical analysis was done using Python 13.0 software. Proportion z-test was used for comparison between the two arms.

At 3 months post Radiotherapy, Radiation Dermatitis was seen in 20 out of 30 patients in the observation arm with Grade I toxicity in 12 patients, Grade II toxicity in 6 patients and Grade III toxicity in 2 patients compared to12 patients having Grade I toxicity who received Curcumin ( $\mathrm{p}=0.038$ ) which was significant. Radiation Cystitis was seen in 21 patients in the observation arm with Grade I Toxicity in 16 Patients, Grade II Toxicity in 4 Patients and Grade IV Toxicity in 1patientcompared to 12patients with Grade I Cystitis in Curcumin arm ( $\mathrm{p}=0.0198)$ which was significant. Radiation Proctitis was seen in 23 patients in the observation arm with Grade I toxicity in 14 Patients and Grade II Toxicity in 9 patients compared to 8 patients with Grade I proctitis and 1 patient with Grade II toxicity in Curcumin arm $(\mathrm{p}=0.001)$ which was significant. There was no evidence of disease in 25 patients and only 5 patients had Stable disease in Curcumin arm compared to 6patients with Stable disease and 4patients with Progressive disease and no evidence of disease was seen in 20 patients in Observation arm. 
Table I: Stage wise Distribution of Patients

\begin{tabular}{|l|c|c|}
\hline STAGE(FIGO- 2018) & NUMBER OF PATIENTS (NO) & PERCENTAGE OF PATIENTS (\%) \\
\hline I & - & - \\
\hline II & 27 & 45 \\
\hline III & 27 & 45 \\
\hline IV & 6 & 10 \\
\hline TOTAL & 60 & 100 \\
\hline
\end{tabular}

Table II: Radiation Therapy Oncology Group (RTOG): BASED TOXICITY (3-months Post-Radiotherapy)

\begin{tabular}{|l|c|c|c|c|c|c|}
\hline TOXICITY & \multicolumn{2}{|c|}{ DERMATITIS } & \multicolumn{2}{c|}{ CYSTITIS } & \multicolumn{2}{c|}{ PROCTITIS } \\
\hline GRADING & $\begin{array}{c}\text { WITH } \\
\text { CURCUMIN }\end{array}$ & $\begin{array}{c}\text { WITHOUT } \\
\text { CURCUMIN }\end{array}$ & $\begin{array}{c}\text { WITH } \\
\text { CURCUMIN }\end{array}$ & $\begin{array}{c}\text { WITHOUT } \\
\text { CURCUMIN }\end{array}$ & $\begin{array}{c}\text { WITH } \\
\text { CURCUMIN }\end{array}$ & $\begin{array}{c}\text { WITHOUT } \\
\text { CURCUMIN }\end{array}$ \\
\hline 1 & 12 & 12 & 12 & 16 & 8 & 14 \\
\hline 2 & - & 6 & - & 4 & 1 & 9 \\
\hline 3 & - & 2 & - & - & - & - \\
\hline 4 & - & - & - & 1 & - & - \\
\hline TOTAL & 12 & 20 & 12 & 21 & 9 & 23 \\
\hline
\end{tabular}

Table III: OUTCOMES OF TREATMENT Post-Radiotherapy

\begin{tabular}{|l|c|c|}
\hline DISEASE STATUS & $\begin{array}{c}\text { WITH } \\
\text { CURCUMIN }\end{array}$ & $\begin{array}{c}\text { WITHOUT } \\
\text { CURCUMIN }\end{array}$ \\
\hline NO EVIDENCE OF DISEASE (NED) & 25 & 20 \\
\hline STABLE DISEASE & 5 & 6 \\
\hline PROGRESSIVE DISEASE & - & 4 \\
\hline TOTAL & 30 & 30 \\
\hline
\end{tabular}
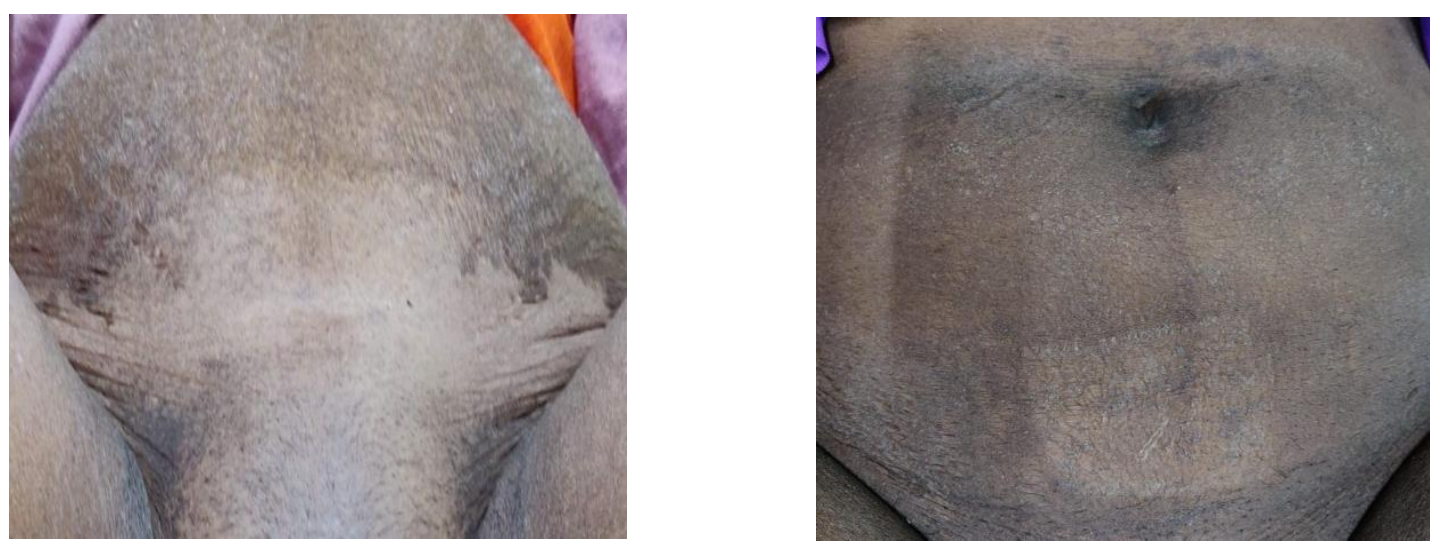

Fig 1: Represents the Grade I Dermatitis and completely healed Skin 1month post RT
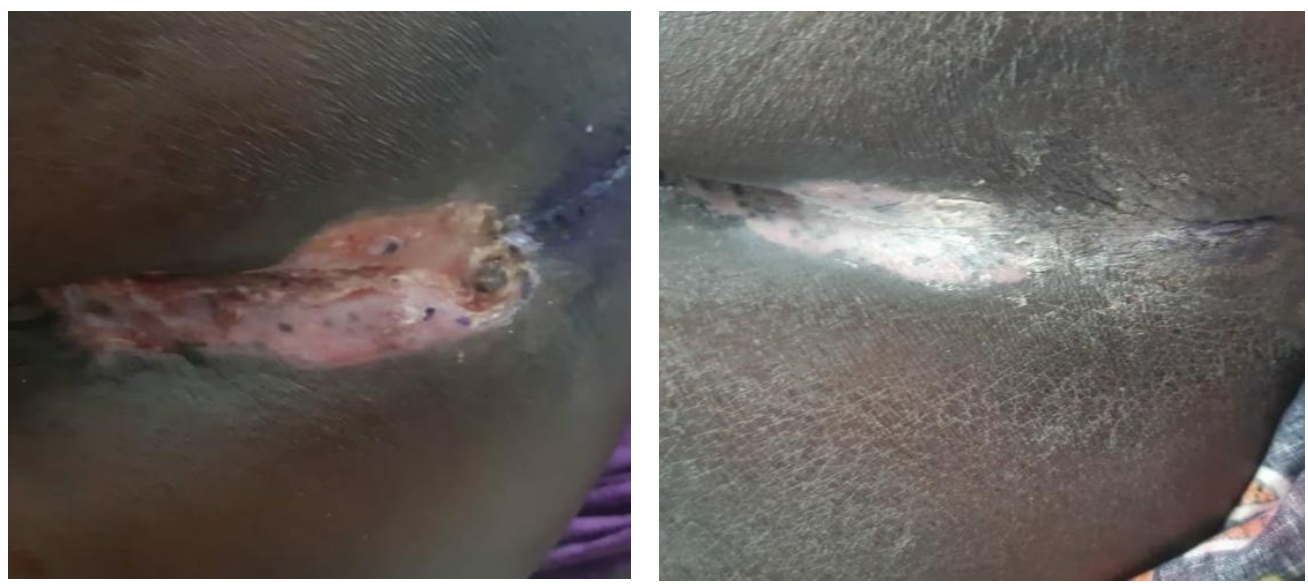

Fig 2: Represents the Grade III Dermatitis and completely healed Skin 3 month post RT. 


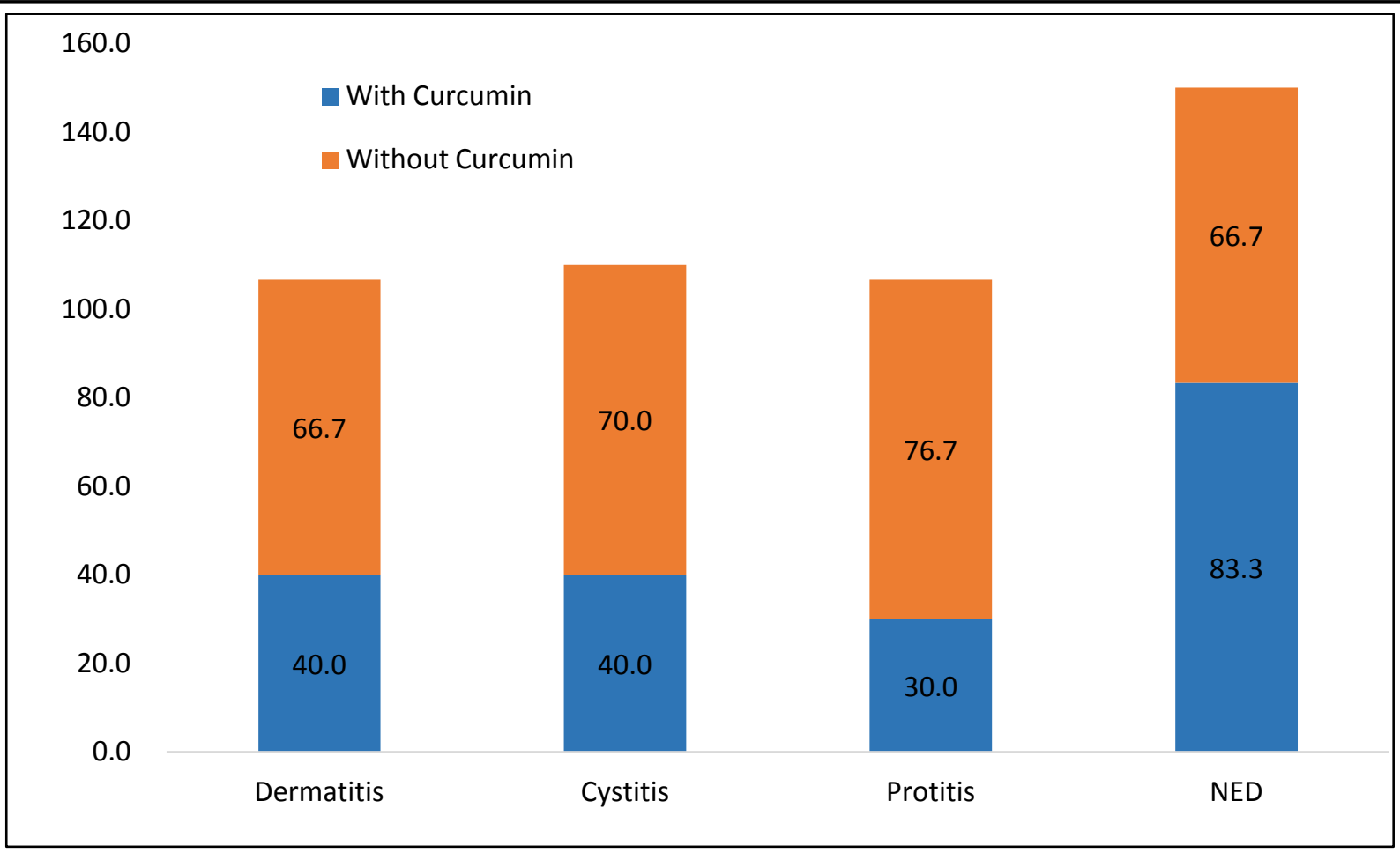

Fig 3: Diagram represents the Toxicity and Disease status in both arms post Radiotherapy.

\section{Discussion}

Radiotherapy in cancer cervix is a "Double Edged sword" in terms of achieving good local control and improving Overall survival, which comes along with complications, contributing to compromised Quality of life. There is a Dose response relationship between dose to Point-A and Local Control, between Bladder dose and Cystitis and between Rectal dose and Proctitis. There is no fixed time duration for development of Radiation Cystitis and Radiation Proctitis. Several factors are associated with complications like Age, Stage, Comorbidities, Modalities of treatment used and Dose received to Bladder and Rectum. Most of the patients being treated need supplements because they have low levels of Vitamins, micronutrients in their body and even their dietary intake is compromised because of complications.

Radiation Dermatitis is the most common side effect of Radiotherapy regardless of site of irradiation. Randomized and double blinded trial of oral curcumin versus placebo in breast cancer patients post radiotherapy. This study favored the usage of curcumin with $p$ value 0.008 along with decreased moist desquamation, which was significant $^{[9]}$.
Roa $\mathrm{S}$ et al studied the effect of curcumin on oral mucositis in Head and Neck Cancer patients receiving radiotherapy and found that it was effective in preventing and reducing radiation induced mucositis. The use of Curcumin as mouth rinse is also beneficial to patients in preventing oral mucositis who receive Chemoradiation in Head and Neck cancer cases. ${ }^{[10,11]}$.

Chendil et al, studied the mechanism of NF-KB pathway and found 3 times lesser surviving prostate cancer cells due to sustained cellular killing and reduced radio resistance, when treated with both Radiotherapy and curcumin ${ }^{[12]}$.

A Turkish study demonstrated that intestinal mucosa was protected to greater degree in rats fed with curcumin, which was proven histopathologically. Bowel toxicity resulting from irradiation during Gynaecologic, Prostate, Gastrointestinal, Pediatric Cancers can be alleviated by the use of curcumin ${ }^{[13]}$.

Montana GS et al evaluated 527 Cancer cervix patients, who received Radiotherapy and found dose response relationship between bladder dose and cystitis. Risk of cystitis was $3-12 \%$ in patients receiving radiation dose ranging from 50 80Gy.The study also showed dose response 
relationship between rectal dose and proctitis. Risk of Proctitis was $2-18 \%$ in patients receiving radiation dose ranging from $50-80 \mathrm{~Gy}^{[14]}$.

During irradiation of the bone marrow in Gynecological malignancy patients, Curcumin protects lymphocytes, the most Radiosensitive blood cell. Thus decreasing Hematological Toxicities. The role of Intensity modulated Radiotherapy has been well established in post operative Cervical cancer cases ${ }^{[15]}$.

MDACC also studied NF-KB modulation although its expression raises after RT. This study also showed that there is decrease in matrix metalloproteinase expression (MMP).This is the enzyme found to be the gateway for metastasis by dissolving bonds of extracellular matrix and also promoting micro environment for growth and spread of cancer cells ${ }^{[16]}$.

Curcumin has good and potent anti-inflammatory effects, which can benefit from Dermatitis, Mucositis, Pnemonitis, Catractogenesis, Neurocognition, Myelosuppression and Secondary tumors, which are the adverse effects of Radiotherapy ${ }^{[17,18]}$.

\section{Conclusions}

Radiation Dermatitis, Cystitis and Proctitis are well known complications of radiation treatment. Early detection and treatment reduces the grade of toxicity and improves Quality of life. Curcumin is a leading armament in naturopathic oncology, which is being used since ages in India. The use of curcumin post radiation definitely mitigates the side effects and thereby improves the therapeutic ratio. The optimal dosage and duration of curcumin post radiotherapy to be administered is still investigational. With the advent of nanocurcumin there is better absorption and bioavailability for enhanced effects of curcumin and improving the quality of life.

The usage of curcumin during radiotherapy is controversial, though it has Radio sensitization effect. Role of prophylactic administration of Curcumin before the onset of disease needs to be answered. Future large randomized trials is required for conclusive evidence for better clinical outcomes. The usage of special formulations like liposomal, conjugated, intravenous and other molecular analogues must also be investigated for better management and control of post radiotherapy complications.

\section{References}

1. Bray F et.al. Global cancer statistics 2018: GLOBOCAN estimates of Incidence and mortality worldwide for 36 Cancers in 185 Countries: CA CANCER J CLIN 2018; 68:394-424.

2. Kidwai Memorial Institute of Oncology, Department of Epidemiology \& Biostatistics, Hospital Based Cancer Registry Annual Report 2015.

3. Goel A, Aggarwal BB: Curcumin, the golden spice from Indian Saffron, is a chemo sensitizer and radio sensitizer for tumors and chemo protector and radio protector for normal organs. Nutr Cancer 2010; 62:919-930.

4. Li M, ZhaNG z, Hill DL, Wang H, Zhang R. Curcumin, a dietary component, has anticancer, chemo sensitization, and radio sensitization effect by down regulating the MDM2 oncogene through the P13K/mTOR/ETS2 pathway. Cancer Res 2007; 67:1988-1996.

5. Sandur SK et.al: Curcumin modulates the radio sensitivity of colorectal cancer cells by suppressing constitutive and inducible NF-Kappa-B activity. Int J Radiat Oncol Biol Phys 2009; 75:534-542.

6. Wei $\mathrm{J}$ et. al. Radiation induced skin reactions: mechanism and treatment. Cancer management and Research 2019:11 167-177.

7. Yang L, LV Y(2012):Possible risk factors associated with radiation Proctitis or Radiation cystitis in patients with cervical carcinoma after radiotherapy. Asian Pac J Cancer Prev, 13, 6252-5. 
8. Mallick S, Madan R, Julka PK, Rath GK. Radiation Induced Cytitis and ProctitisPrediction, Assessment and Management. Asian Pac J Cancer Prev .16(14),55895594.

9. Ryan JL, Heckler CE, Ling $M$ et al. Curcumin for Radiation dermatitis: A randomized, double-blind, placebocontrolled clinical trial of thirty breast cancer patients. Radiat Res.2013; 180(1):34-43.

10. Rao S et.al: The Indian spices Turmeric delays and Mitigates Radiation-Induced Oral Mucositis in Patients Undergoing Treatment for Head and Neck Cancer: An Investigational study. Integr Cancer Ther 2014; 13:201-210.

11. Patil K, Gudledgud MV, Kulkarani PK, Keshari D, Tayal S: Use of curcumin mouth rinse in Radio-Chemotherapy Induced Oral Mucositis Patients: A Pilot study . J Clin Diag Rs 2015; 9:59-62.

12. Chendil D, Ranga RS, Meigooni D, Sathishkumar S, Ahmed MM: Curcumin confers radio sensitizing effect in prostate cancer cell line PC-3.Oncogene 2004;23:1599-1607.

13. Akpolat M,Kanter M,Uzal MC.Protective effects of curcumin against gamma radiation-induced ileal mucosal damage. Arch Toxicol 2009; 83:609-617(Turkish Study).

14. Montana GS, Fowler WC (1989). Carcinoma of the cervix: Analysis of Bladder and rectal Radiation dose and complications. Int J Radiat Oncolo Biol Phys, 16, 95-100.

15. Zelefsky MJ, Levin EJ, Hunt $M$ et al (2008): Incidence of late rectal and urinary toxicities after three-dimensional conformal radiotherapy and intensitymodulated Radiotherapy for localized prostate cancer. Int J Radiat Oncol Biol Phys, 70, 1124-9.
16. Kunnumakkara AB et.al: Curcumin sensitizes human colorectal cancer xenografts in nude mice to gammaradiation by targeting nuclear factor kappa B-regulated gene products. Clin Cancer Res 2008; 14:2128-2136.

17. Jagetia GC. Radioprotection and Radio sensitization by curcumin. Adv Exp Med Biol 2007; 595:301-320.

18. Verma V: Relationship and interactions of curcumin with radiation therapy. World $\mathrm{J}$ Clin Oncol 2016; 7(3):275-283. 